\title{
An Improved Nonlinear Grey Bernoulli Model Based on the Whale Optimization Algorithm and Its Application
}

\author{
Jianming Jiang $\mathbb{D},{ }^{1}$ Ting Feng $\mathbb{D},{ }^{1}$ and Caixia Liu $\mathbb{D}^{2}$ \\ ${ }^{1}$ School of Mathematics and Statistics, Baise University, Baise, China \\ ${ }^{2}$ Institute of EduInfo Science \& Engineering, Nanjing Normal University, Nanjing, China \\ Correspondence should be addressed to Caixia Liu; cxsqz@126.com
}

Received 8 October 2020; Revised 27 January 2021; Accepted 28 February 2021; Published 11 March 2021

Academic Editor: Leonid Shaikhet

Copyright (C) 2021 Jianming Jiang et al. This is an open access article distributed under the Creative Commons Attribution License, which permits unrestricted use, distribution, and reproduction in any medium, provided the original work is properly cited.

In order to improve the prediction performance of the existing nonlinear grey Bernoulli model and extend its applicable range, an improved nonlinear grey Bernoulli model is presented by using a grey modeling technique and optimization methods. First, the traditional whitening equation of nonlinear grey Bernoulli model is transformed into its linear formulae. Second, improved structural parameters of the model are proposed to eliminate the inherent error caused by the leap jumping from the differential equation to the difference one. As a result, an improved nonlinear grey Bernoulli model is obtained. Finally, the structural parameters of the model are calculated by the whale optimization algorithm. The numerical results of several examples show that the presented model's prediction accuracy is higher than that of the existing models, and the proposed model is more suitable for these practical cases.

\section{Introduction}

Professor Deng [1] originally proposed the grey system theory to solve the uncertain system with partially known and partially unknown information. As a crucial branch of the grey system theory, it has been widely used to address numerous real-world problems owing to its effectiveness, such as electricity prediction [2-4], energy prediction $[5,6]$, and tourism prediction [7]. In these models, a common characteristic is that they do not require a large number of observations (not less than 4). It has attracted considerable interests of researchers because it is difficult, even impossible, to collect enough data to build the traditional models, including linear [8] or nonlinear regression models [9], autoregressive integrated moving average model [10] and its extensive versions [11], support vector machine [12], and artificial neural network [13].

Generally speaking, the development of discipline also benefits from practical applications. In the past three decades, various grey models have been emerged rapidly according to practical applications. For example, Xie and Liu [14] investigated the discrete grey model and analyzed the traditional grey model's connection. Wu et al. [15] investigated the grey model with fractional order accumulation that made the grey model more flexible. For the purpose of considering the effects of related factors on the behavioral system, Tien [16] initially proposed a novel grey model called GM $(1, \mathrm{n})$ in which the " $\mathrm{n}$ " stands for the $n-1$ driving variable. More recently, Wang et al. [17] presented a data-grouping approach-based grey modeling method to predict quarterly hydropower production in China. Subsequently, they proposed a seasonal grey model based on the accumulation operators for forecasting the seasonal electricity consumption of China [18]. Zeng et al. [19] predicted the sequence of ternary interval numbers using a novel multivariable grey model. Ma et al. [20] raised a conformable fractional grey system model; he also investigated the novel fractional time-delayed grey model with grey wolf optimizer [21]. A large number of related research studies emerge continuously. Zeng et al. [22] presented a new-structure grey Verhulst model for predicting China's tight gas production. In the model, they deduced the time-response function and an initial value optimization method. The same year, they proposed another new- 
structure grey Verhulst model by introducing a new nonhomogeneous exponential function [23]. The model solved the problem of displacement substitution of parameters and optimization of initial values.

The metaheuristic algorithm is a strategy to solve the optimal or satisfactory solution of complex optimization problems, and it is derived from the behavior of biological systems and/or physical systems in nature [24]. The common metaheuristic algorithm includes simulated annealing algorithm [25], genetic algorithm [26], particle swarm optimization algorithm [27], and ant colony optimization algorithm [28]. For example, the simulated annealing algorithm was first used by Kirpatrick et al. [25] for combinatorial optimization problems, which overcomes the shortcoming of the hill-climbing method (HC) and is easy to fall into local solution. Yldz et al. [29] studied metaheuristic methods and proved that Henry gas solubility optimization algorithm can be used for solving shape optimization problems. The main development directions of the metaheuristic algorithm can be divided into three classes. The first is to combine with other algorithms to form a new hybrid algorithm to give full play to their characteristics. Yildiz et al. [30] presented a hybrid optimization algorithm combining the Nelder-Mead local search algorithm with the Harris hawks optimization algorithm for solving a milling manufacturing optimization problem. Similarly, a hybrid optimization algorithm based on the Nelder-Mead local search algorithm and whale optimization algorithm was proposed to accelerate global convergence speed of the whale algorithm [31], and the algorithm optimized the processing parameters in manufacturing processes. The second is to seek a new metaheuristic algorithm for optimization of complex problems in the real world from the mechanism of biological evolution. For example, Wang et al. [32] proposed a monarch butterfly optimization algorithm. The third is to improve the existing algorithms by introducing new mechanics or strategies. Hammou et al. [33] improved the particle swarm optimization algorithm with a strategy based on cooperation and hierarchization concepts for the updating of the best personal positions of particles. In recent years, metaheuristic algorithms are used in grey models for finding the optimal parameter solutions. Zhang et al. [34] optimized the background value weighting coefficients of the grey model using the genetic algorithm. In [35], a multiobjective grey wolf optimizer was used to optimize the kernel-based nonlinear extension of the Arps decline model to ensure both prediction stability and accuracy. Wu et al. [36] used the particle swarm optimization algorithm to search optimal system parameters of the nonlinear grey Bernoulli model.

This study focuses on improving the nonlinear grey Bernoulli model, which was initially proposed by Chen [37] and abbreviated as $\operatorname{NGBM}(1,1)$. As is known, $\operatorname{NGBM}(1,1)$ has been widely used in many problems with nonlinear characteristics and extended to general versions [38]. However, there are still spaces to improve its accuracy. The root cause of loss of information in the conversion of the grey differential equation to the grey difference equation is proposed in the paper [39]. Following the thought of $\mathrm{Ma}$ et al. [7], the model parameters of the $\operatorname{NGBM}(1,1)$ model are optimized to better match these two equations to reduce prediction error. The main contributions of this paper are drawn as follows: (1) the grey differential equation is transformed into linear form rather than sharing the same form to the traditional NGBM $(1,1)$ model; $(2)$ the optimized parameters are constructed and the whale optimization algorithm (WOA) is used to search for the optimal power index; (3) three cases are employed to verify the effectiveness of INGBM $(1,1)$.

The rest of this paper is organized as follows: Section 2 briefly describes the NGBM $(1,1)$ model and obtains the "linear" solution to the $\operatorname{NGBM}(1,1)$ model. In Section 3, the $\operatorname{NGBM}(1,1)$ model with improved parameters is deduced in detail. Section 4 provides two real-world examples to validate the effectiveness of the proposed model. Section 5 applies INGBM $(1,1)$ to predict the number of R\&D institutions of higher education in China to reveal the forecasting ability of $\operatorname{INGBM}(1,1)$, and the main conclusions are listed in the final section.

\section{Description of the Nonlinear Grey Bernoulli Model}

The nonlinear grey Bernoulli model (NGBM $(1,1)$ ), originally proposed by Chen [37], has wide applications, especially in solving nonlinear problems. However, this model still has some drawbacks that impair the prediction accuracy of NGBM $(1,1)$. This section is to analyze the root reason and propose a novel method to reduce the modeling bias. First, a brief description of NGBM $(1,1)$ is introduced. Additionally, a "linear" solution to the whitening equation of $\operatorname{NGBM}(1,1)$ is proposed to make the parameter optimization more simplified.

2.1. The Traditional Solution to the Nonlinear Grey Bernoulli Model. Assume

$$
X^{(0)}=\left\{x^{(0)}(1), x^{(0)}(2), \ldots, x^{(0)}(n)\right\},
$$

to be a nonnegative series, and then the first-order accumulative generating operator (1-AGO) series is

$$
X^{(1)}=\left\{x^{(1)}(1), x^{(1)}(2), \ldots, x^{(1)}(n)\right\},
$$

where $x^{(1)}(k)=\sum_{i=1}^{k} x^{(0)}(i)$.

The equation

$$
\frac{\mathrm{d} x^{(1)}(t)}{\mathrm{d} t}+a x^{(1)}(t)=b\left[x^{(1)}(t)\right]^{\gamma}
$$

is called the whitening equation of nonlinear grey Bernoulli model and $n$, regarded as the power index, cannot be equal to one. With the two-point trapezoidal formula, the discrete difference equation can be written as

$$
x^{(0)}(k)+a z^{(1)}(k)=b\left[z^{(1)}(k)\right]^{\gamma},
$$

where $z^{(1)}(k)$ represents the background value and is obtained as 


$$
\begin{gathered}
z^{(1)}(k)=0.5\left(x^{(1)}(k)+x^{(1)}(k-1)\right) \\
\text { Let } B=\left[\begin{array}{cc}
-z^{(1)}(2) & z^{(1)}(2)^{\gamma} \\
-z^{(1)}(3) & z^{(1)}(3)^{\gamma} \\
\vdots & \vdots \\
-z^{(1)}(n) & z^{(1)}(n)^{\gamma}
\end{array}\right], \quad Y=\left[x^{(0)}(2), x^{(0)}(3),\right. \\
\left.\ldots, x^{(0)}(n)\right]^{T} \text {. }
\end{gathered}
$$

The model parameters can be estimated by the least-squares method and shown that

$$
(a, b)^{T}=\left(B^{T} B\right)^{-1} B^{T} Y
$$

Therefore, the solution to equation (3) with $x^{(1)}(1)=$ $x^{(0)}(1)$ is

$$
\widehat{x}^{(1)}(k)=\left(\left(x^{(0)}(1)^{1-\gamma}-\frac{b}{a}\right) e^{-a(1-\gamma)(k-1)}+\frac{b}{a}\right)^{(1 /(1-\gamma))} .
$$

Using the firs-order inverse accumulative generating operator (1-IAGO), the simulated values of $X^{(0)}, \widehat{X}^{(0)}$, is

$$
\widehat{x}^{(0)}(k)=\widehat{x}^{(1)}(k)-\widehat{x}^{(1)}(k-1) .
$$

\subsection{The "Linear" Solution to Nonlinear Grey Bernoulli Model.} This section transforms the whitening equation of the nonlinear grey Bernoulli model $(\operatorname{NGBM}(1,1))$ into the linear formulation, rather than directly solving the whitening equation. That is, it does not share the same pattern as the traditional grey model. The detailed computational process can be depicted as follows.

Analogously to Section 2.1, both sides of whitening equation (3) are multiplied by $x^{(1)}(t)^{-\gamma}$, and then

$$
x^{(1)}(t)^{-\gamma} \frac{\mathrm{d} x^{(1)}(t)}{\mathrm{d} t}+a x^{(1)}(t)^{1-\gamma}=b .
$$

Set $y^{(1)}(t)=x^{(1)}(t)^{1-\gamma}$; furthermore,

$$
\frac{\mathrm{d} y^{(1)}(t)}{\mathrm{d} t}=(1-\gamma) x^{(1)}(t)^{-\gamma} \frac{\mathrm{d} x^{(1)}(t)}{\mathrm{d} t} .
$$

Thereby, equation (10) can be written as

$$
\frac{\mathrm{d} y^{(1)}(t)}{\mathrm{d} t}+a(1-\gamma) y^{(1)}(t)=b(1-\gamma)
$$

which is called the linearization of the $\operatorname{NGBM}(1,1)$ model. Moreover, it easily yields the discrete form by using the twopoint trapezoidal formula as follows:

$$
y^{(0)}(k)+a(1-\gamma) z_{y}^{(1)}(k)=b(1-\gamma),
$$

where $z_{y}^{(1)}(k)=0.5\left(y^{(1)}(t)+y^{(1)}(t-1)\right)$. If

$$
\chi=\left[\begin{array}{cc}
-(1-\gamma) z_{y}^{(1)}(2) & (1-\gamma) \\
-(1-\gamma) z_{y}^{(1)}(3) & (1-\gamma) \\
\vdots & \vdots \\
-(1-\gamma) z_{y}^{(1)}(n) & (1-\gamma)
\end{array}\right] \text { and }
$$

$Y_{R}=\left[y^{(0)}(2), y^{(0)}(3), \ldots, y^{(0)}(n)\right]^{T}$, the parameters can be estimated by the least-squares method and shown that

$$
\left(\begin{array}{l}
a \\
b
\end{array}\right)=\left(\chi^{T} \chi\right)^{-1} \chi^{T} Y_{R}
$$

After estimating the model parameters, the whitening equation, equation (11), is resolved. Multiply both sides in equation (11) by the integrating operator $e^{a(1-\gamma) t}$ :

$$
\frac{\mathrm{d}\left[e^{a(1-\gamma) t} y^{(1)}(t)\right]}{\mathrm{d} t}=b(1-\gamma) e^{a(1-\gamma) t} .
$$
$[1, t]:$

Integrate both sides in equation (14) over the interval

$$
\int_{1}^{t} d\left[e^{a(1-\gamma) \tau} y^{(1)}(\tau)\right]=\int_{1}^{t} b(1-\gamma) e^{a(1-\gamma) \tau} \mathrm{d} \tau,
$$

and which is also

$$
y^{(1)}(t)=\left(y^{(0)}(1)-\frac{b}{a}\right) e^{-a(1-\gamma)(t-1)}+\frac{b}{a} .
$$

According to 1 -AGO and $y^{(1)}(t)=x^{(1)}(t)^{1-\gamma}$,

$$
\left\{\begin{array}{l}
\hat{x}^{(0)}(k)=\widehat{x}^{(1)}(k)-\widehat{x}^{(1)}(k-1), \\
\hat{x}^{(1)}(k)=\hat{y}^{(1)}(k)^{(1 /(1-\gamma))} .
\end{array}\right.
$$

The solution of the $\operatorname{NGBM}(1,1)$ model, either in linearization or in nonlinearization, is essentially approximate because the conversion of equations (11) and (12) is based on two-point trapezoidal formula regarded as an approximate method. It implies that the "misplaced replacement" of the model parameters will cause the following: (i) the difference grey equation does not match with the differential grey equation because model parameters have different meanings in these equations; (ii) the prediction model is not satisfied in most situations. It indicates the performance of the NGBM $(1,1)$ model must be improved. In other words, the model parameters should be optimized to better match equations (11) and (12) and to increase the forecasting ability of the NGBM $(1,1)$ model.

\section{Parameter Optimization of Nonlinear Grey Bernoulli Model}

The whitening equation parameters, $a, b$ and power index $\gamma$, are important parameters of the nonlinear grey Bernoulli model. In this section, the parameters are calculated.

3.1. Whitening Equation Parameter Calculation. The optimized parameters, $a$ and $b$, are denoted as $p$ and $q$ for simplicity. The optimized parameters are substituted into the time-response function, and the following equation is obtained:

$$
\widehat{y}^{(1)}(t)=\left(y^{(0)}(1)-\frac{q}{p}\right) e^{-p(1-\gamma)(t-1)}+\frac{q}{p} .
$$

Equation (18) is substituted into the left-hand side in equation (4): 


$$
\begin{aligned}
L(t) & =\left[\widehat{y}^{(1)}(t)-\hat{y}^{(1)}(t-1)\right]+\frac{a}{2}(1-\gamma)\left[\hat{y}^{(1)}(t)+\widehat{y}^{(1)}(t-1)\right] \\
& =\left(1+\frac{a}{2}(1-\gamma)\right)\left[\left(y^{(0)}(1)-\frac{q}{p}\right) e^{-p(1-\gamma)(t-1)}+\frac{q}{p}\right]-\left(1-\frac{a}{2}(1-\gamma)\right)\left[\left(y^{(0)}(1)-\frac{q}{p}\right) e^{-p(1-\gamma)(t-2)}+\frac{q}{p}\right] \\
& =\left[1+\frac{a}{2}(1-\gamma)-\left(1-\frac{a}{2}(1-\gamma)\right) e^{p(1-\gamma)}\right]\left(y^{(0)}(1)-\frac{b}{a}\right) e^{-p(1-\gamma)(t-1)}+a(1-\gamma) \frac{q}{p}
\end{aligned}
$$

According to equation (4), the left-hand side $L(t)$ should be equal to the right-hand side $R(t)$; that is, $L(t)-R(t)=0$. Therefore,

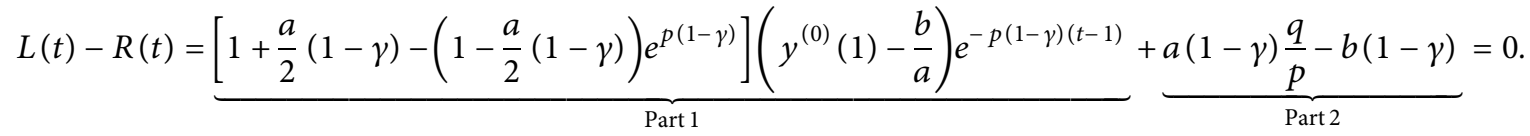

It is easy to find that Part 1 and 2 both are equal to zero in equation (20); hence,

$$
\begin{aligned}
{\left[1+\frac{a}{2}(1-\gamma)-\left(1-\frac{a}{2}(1-\gamma)\right) e^{p(1-\gamma)}\right] } & =0 \Rightarrow p=\frac{1}{1-\gamma} \ln \left(\frac{1+(a / 2)(1-\gamma)}{1-(a / 2)(1-\gamma)}\right) \\
a(1-\gamma) \frac{q}{p}-b(1-\gamma) & =0 \Rightarrow q=\frac{p b}{a}
\end{aligned}
$$

By doing so, the optimized parameters $p$ and $q$ can be estimated. Moreover, it is obviously believed that the optimized parameters can better match the differential equation and the difference equation and reduce the prediction error. For simplicity, $\operatorname{NGBM}(1,1)$ with the improved parameters is abbreviated as $\operatorname{INGBM}(1,1)$ in this study.

\subsection{Power Index Estimation Based on the Whale Optimization} Algorithm. In the above descriptions, the power index $\gamma$ is assumed to be known. However, the power index is always changeable in a different situation that requires flexible adjusting over given datasets. To solve this problem, an intelligent algorithm, whale optimization algorithm, shorted for WOA, is employed to automatically determine the power index.

Based on the humpback whale's hunting behavior that recognizes the location of prey and encircles them, Mirjalili and Lewis designed the WOA [40]. In this optimizer, assume the current best candidate solution (search agent) to be the target prey or be near the optimum. Once the best search agent is defined, the other search agents will update their positions towards the best search agent:

(i) In this behavioral system, they update their position by

$$
\begin{aligned}
\vec{D} & =\left|\vec{C} \overrightarrow{X^{*}}-\vec{X}(t)\right|, \\
\vec{X}(t+1) & =\overrightarrow{X^{*}}(t)-\vec{A} \cdot \vec{D},
\end{aligned}
$$

where $t$ represents the current iteration, $\vec{X}^{*}(t)$ is the current best agent, and $\vec{D}=\left(D^{1}, D^{2}, \ldots\right.$, $\left.D^{d}\right), j=1,2, \ldots, d$ denotes the length of the individual whale approaching the current best search agent in $j^{\text {th }}$ spatial position. In particular, the coefficient vector $\vec{A}$ and $\vec{C}$ are defined as

$$
\begin{aligned}
& \vec{A}=2 a \cdot r-a, \\
& C=2 \cdot r,
\end{aligned}
$$

where $r$ is a random number generated from $[0,1]$ and $a$ is called convergence factor that linearly decreases from 2 to 0 . That is,

$$
a=2-\left(\frac{2 t}{t_{\max }}\right)
$$

(ii) A spiral equation is also designed between the position of whale and prey to mimic the helixshaped movement of humpback whales: 


$$
\vec{X}(t+1)=\vec{D}^{\prime} \cdot e^{b l} \cdot \cos (2 \pi l)+\vec{X}^{*}(t),
$$

where $\vec{D}^{\prime}=\left|\vec{X}^{*}(t)-\vec{X}(t)\right|$ and implies the distance of the $i^{\text {th }}$ whale to the prey, $b$ is a constant for fixing the shape of the logarithmic spiral, and $l$ is a random number and $l \in[-1,1]$.

(iii) In addition, humpback whales also search for prey in a random way according to the position of each other. This behavior is written as the following mathematical expression:

$$
\begin{gathered}
\vec{D}=\left|\vec{C} \cdot \vec{X}_{\text {rand }}-\vec{X}\right|, \\
\vec{X}(t+1)=\vec{X}_{\text {rand }}(t)-A \cdot \vec{D}_{\text {rand }},
\end{gathered}
$$

where $\vec{X}_{\text {rand }}$ is a random position chosen from the current position. For clearness, the detailed steps of the algorithm based on WOA to find the optimal $\gamma$ are listed as follows:

Step 1: set algorithm parameters $N$, $\operatorname{dim}$, and $t_{\max }$. Step 2: initialize the whales' population $X_{i}(i=1,2, \ldots, n)$.

Step 3: calculate the fitness of each search agent $f\left(\vec{X}_{i}\right)$.

Step 4: update $a, A$, and $C$ according to equations (24)-(26).

Step 5: generate a random number $p$ in $[0,1]$. If $p \geq 0.5$, update the position of the current search agent by equation (27). If $p<0.5$ and $|A| \geq 1$, update the position of the current search agent by equation (29). If $p<0.5$ and $|A|<1$, update the position of the current search agent by equation (22).

Step 6: return to Step 3, until the optimal value $\gamma$ is found.

Note that the fitness function, $f\left(\vec{X}_{i}\right)$, as usual, is often defined as an objective function, MAPE, and shown in the next section. Moreover, the flowchart of the $\operatorname{INGBM}(1,1)$ model is graphed in Figure 1 for clearness.

\section{Validation of the Nonlinear Grey Bernoulli Model}

This section provides two examples to demonstrate the efficacy of the proposed model comparing with three competing models, including the GM $(1,1)$, DGM $(1,1)$, NGBM $(1,1)$, and $\operatorname{ONGBM}(1,1)$. Additionally, to evaluate the prediction accuracy of these grey models, the mean absolute percentage error (MAPE) and root mean square error (RMSE) are applied to measure the level of prediction performance, which are defined as

$$
\begin{aligned}
& \text { MAPE }=\frac{1}{n-1} \sum_{i=2}^{n} \frac{\left|\hat{x}^{(0)}(k)-x^{(0)}(k)\right|}{x^{(0)}(k)} \times 100 \%, \\
& \text { RMSE }=\sqrt{\frac{1}{n-1} \sum_{i=2}^{n}\left(\hat{x}^{(0)}(k)-x^{(0)}(k)\right)^{2}} .
\end{aligned}
$$

The grade of the prediction performance is depicted by Lewis [41] using the criteria for MAPE and listed in Table 1.

Case 1. Forecasting education-in-practice-intensive university: the example from paper [42] is used to test for efficacy and applicability of the grey model. The data from 1 to 7 are used to build different grey models, and the final data are used to test for the prediction accuracies of these models. Accordingly, the five models' parameters are listed in Table 2 , and especially parameter values of the proposed model by WOA are graphed in Figure 2.

Consequently, the simulation and prediction results are shown in Table 3.

Case 2. Forecasting subway passenger: the data sets of example from paper [43] are empirically broken down into two groups: the data from 2005 to 2012 are used to build five grey models, and the other data are used to test for the prediction accuracies of these models.

First of all, the parameter values of the five grey models are computed in Table 4. Moreover, the track of searching for the optimal nonlinear parameter of the INGBM $(1,1)$ model using WOA is graphed in Figure 3.

Furthermore, the simulation and prediction results are shown in Table 5.

In Tables 1-5, the desired conclusions can be drawn as follows:

(1) In case 1 , the INGBM $(1,1)$ model has a better prediction performance than that of other grey models whether in simulated or predicted period because of its lowest MAPE values which are 1.05\% and $12.78 \%$, respectively. Incidentally, it is notable that the MAPE values of all models increase to more than $10 \%$, which are $19.17 \%, 18.86 \%, 14.17 \%$, $15.44 \%$, and $12.78 \%$, respectively. This indicates that these models do not work quite well in this case. Nevertheless, the proposed model, INGBM $(1,1)$, outperforms these models. It can be seen that in this case, the fitting errors of all models are relatively small, while the prediction errors are relatively large, which indicates that the model has overfitted a little on this data set. The issue can be overcome by adding some penalty terms.

(2) In case 2, the five grey models' MAPE values are $2.61 \%, 2.67 \%, 2.23 \%, 2.23 \%$, and $2.19 \%$ in the simulated period, respectively. According to the criteria 


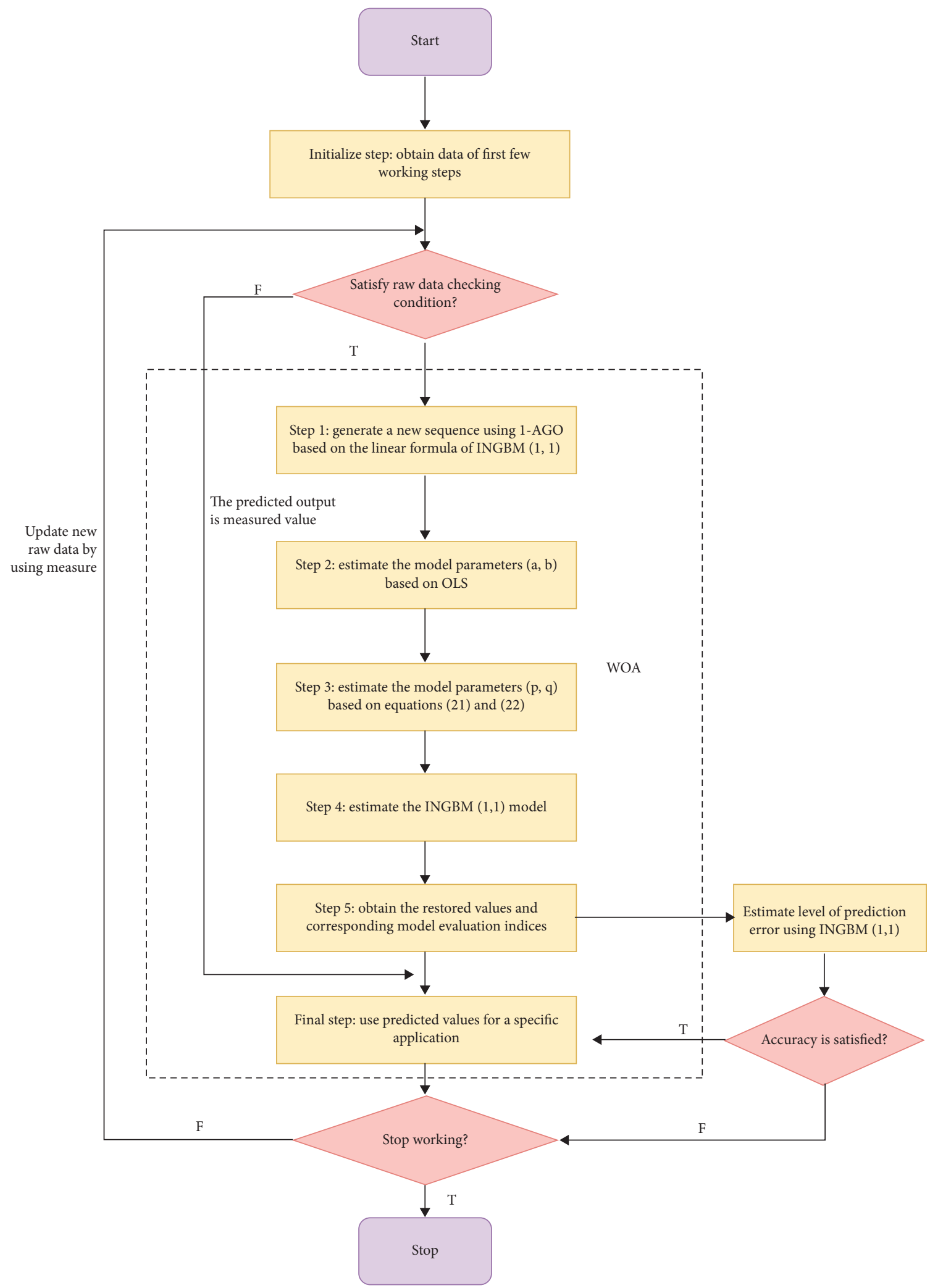

FIGURE 1: Flowchart of the INGBM $(1,1)$ model.

TABLE 1: The criteria for MAPE proposed by Lewis.

\begin{tabular}{lcccc}
\hline MAPE & $<10$ & $10 \sim 20$ & $20 \sim 50$ & $>50$ \\
\hline Forecasting ability & Excellent & Good & Reasonable & Weak \\
\hline
\end{tabular}


TABle 2: Parameter values for five grey models.

\begin{tabular}{lccccccc}
\hline Parameters & $a$ & $b$ & $\zeta$ & $\gamma$ & $\beta_{1}$ & $\beta_{2}$ & $p$ \\
\hline GM $(1,1)$ & -0.164 & 748.161 & 0.500 & & & & \\
DGM $(1,1)$ & & & & & 1.179 & 817.126 & \\
NGBM $(1,1)$ & -0.213 & 19390.960 & 0.500 & -0.440 & & & \\
ONGBM $(1,1)$ & -0.211 & 20938.930 & 0.460 & -0.450 & & & -0.222 \\
INGBM $(1,1)$ & -0.220 & 39847.500 & 0.500 & -0.542 & & 42037.640 \\
\hline
\end{tabular}

Note: (represents the weighted parameter of background value and it is taken as 0.5 generally. It is, however, recommended to search for the optimal value in ONGBM $(1,1)$. In addition, $\beta_{1}$ and $\beta_{2}$ are parameters of DGM $(1,1)$ in this case.

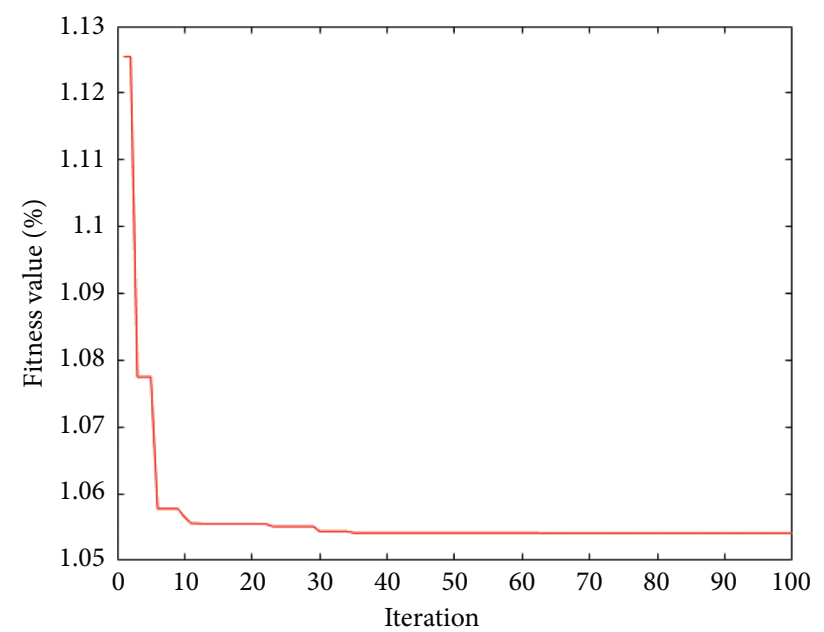

FIgure 2: The track of searching for the optimal power index by WOA.

TABLE 3: Simulated and predicted results by different grey models.

\begin{tabular}{|c|c|c|c|c|c|}
\hline Actual data & GM $(1,1)$ & $\operatorname{DGM}(1,1)$ & $\operatorname{NGBM}(1,1)$ & $\operatorname{ONGBM}(1,1)$ & $\operatorname{INGBM}(1,1)$ \\
\hline \multicolumn{6}{|l|}{1033} \\
\hline 1105 & 997.76 & 1001.69 & 1105.03 & 1105.55 & 1105.02 \\
\hline 1199 & 1176.05 & 1180.66 & 1178.47 & 1173.25 & 1158.90 \\
\hline 1330 & 1386.20 & 1391.61 & 1352.46 & 1342.52 & 1330.20 \\
\hline 1583 & 1633.90 & 1640.25 & 1599.05 & 1583.71 & 1581.54 \\
\hline 1882 & 1925.86 & 1933.31 & 1920.67 & 1898.57 & 1914.91 \\
\hline 2370 & 2269.99 & 2278.73 & 2328.86 & 2298.04 & 2343.38 \\
\hline RMSE & 70.36 & 69.58 & 26.99 & 32.33 & 23.81 \\
\hline MAPE & 4.27 & 4.28 & 1.37 & 1.18 & 1.05 \\
\hline 3310 & 2675.62 & 2685.87 & 2849.94 & 2798.78 & 2886.89 \\
\hline RMSE & 634.38 & 624.13 & 469.06 & 511.22 & 423.11 \\
\hline MAPE & 19.17 & 18.86 & 14.17 & 15.44 & 12.78 \\
\hline
\end{tabular}

TABLE 4: Parameter values for five grey models.

\begin{tabular}{lccccccc}
\hline Parameters & $a$ & $b$ & $\zeta$ & $\gamma$ & $\beta_{1}$ & $\beta_{2}$ & $p$ \\
\hline GM $(1,1)$ & -0.155 & 464.614 & 0.500 & & & & \\
DGM $(1,1)$ & & & & & 1.168 & 503.98 & \\
NGBM $(1,1)$ & -0.138 & 247.382 & 0.500 & 0.094 & & & \\
ONGBM $(1,1)$ & -0.137 & 238.140 & 0.510 & 0.100 & & & \\
INGBM $(1,1)$ & -0.133 & 216.435 & 0.500 & -0.115 & & 0.133 \\
\hline
\end{tabular}




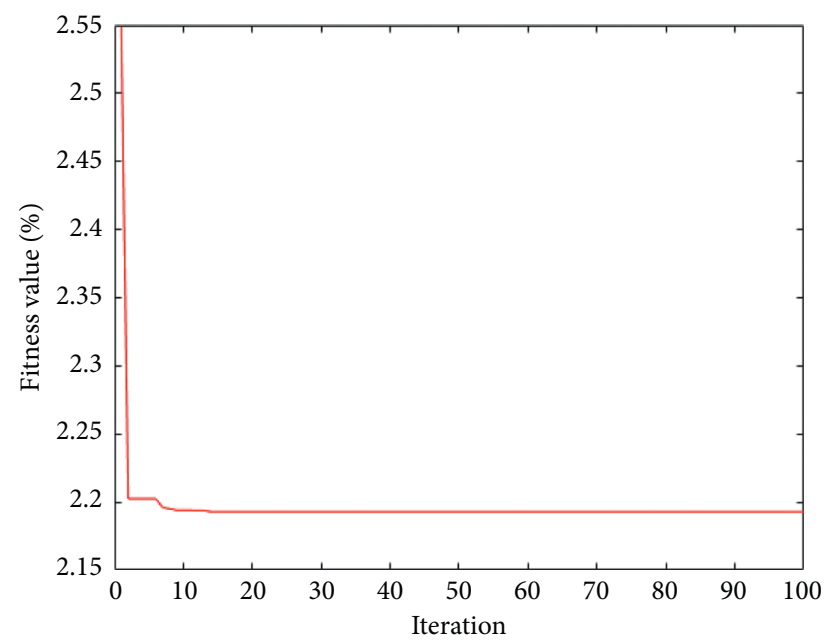

FIGURE 3: The track of searching for the optimal power index by WOA.

TABLE 5: Simulated and predicted results by different grey models.

\begin{tabular}{|c|c|c|c|c|c|}
\hline Actual data & GM $(1,1)$ & DGM $(1,1)$ & NGBM $(1,1)$ & $\operatorname{ONGBM}(1,1)$ & INGBM $(1,1)$ \\
\hline \multicolumn{6}{|l|}{467.63} \\
\hline 561.41 & 581.03 & 582.55 & 561.45 & 561.29 & 561.41 \\
\hline 705.23 & 678.50 & 680.42 & 674.36 & 675.50 & 678.27 \\
\hline 812.94 & 792.33 & 794.75 & 796.23 & 798.30 & 802.45 \\
\hline 895.20 & 925.26 & 928.28 & 932.31 & 935.07 & 939.61 \\
\hline 1069.41 & 1080.48 & 1084.25 & 1086.26 & 1089.52 & 1093.49 \\
\hline 1305.30 & 1261.74 & 1266.42 & 1261.54 & 1265.08 & 1267.40 \\
\hline 1463.00 & 1473.42 & 1479.20 & 1461.75 & 1465.33 & 1464.76 \\
\hline RMSE & 25.51 & 25.30 & 26.21 & 25.96 & 26.26 \\
\hline MAPE & 2.61 & 2.67 & 2.23 & 2.23 & 2.19 \\
\hline 1705.37 & 1720.60 & 1727.73 & 1690.87 & 1694.21 & 1689.28 \\
\hline 1868.94 & 2009.25 & 2018.02 & 1953.39 & 1956.15 & 1945.04 \\
\hline 1931.75 & 2346.33 & 2357.08 & 2254.40 & 2256.14 & 2236.66 \\
\hline RMSE & 165.53 & 170.56 & 126.18 & 127.03 & 118.94 \\
\hline MPAE & 4.27 & 4.47 & 3.15 & 3.16 & 2.97 \\
\hline
\end{tabular}

for MAPE value listed in Table 1, it is easy to find that these models can effectively make predictions because of the low MAPE values. The proposed model has a smaller value that indicates higher accuracy. As is known, a favorable predictor performs well in the simulated period and satisfies prediction accuracy in the verifying period. Herein, the proposed model still is better than other grey models because of its lower MAPE value again in the predicted period. In this case, the fitting error and prediction error of all the models are small, which shows that no fitting has occurred. More, the nonlinear model $\operatorname{NGBM}(1,1)$, $\operatorname{ONGBM}(1,1)$, and INGBM $(1,1))$ performs better than the linear model $(\operatorname{GM}(1,1)$ and $\operatorname{DGM}(1,1))$, which proves that the nonlinear grey model can well capture the nonlinear characteristics of the data.

In cost-effectiveness, the grey model is a kind of model solving small sample modeling, so the time consumption is usually very small. For example, in case 1, the time cost of GM $(1,1)$, DGM $(1,1)$, NGBM $(1,1)$, and INGBM $(1,1)$ is 0.1638 s, 0.1489 s, 0.1744 s, and 0.1862 s, respectively. All the time costs are less than $1 \mathrm{~s}$ and within the allowable range. In summary, the INGBM $(1,1)$ model can enhance the prediction accuracy of the traditional $\operatorname{NGBM}(1,1)$ model by optimizing the model parameters. Furthermore, the proposed model is applied to analyze the practical application.

\section{Application}

Universities play an irreplaceable role in the process of building a strong country in the field of science and technology in China, as the core department for cultivating talent and achieving technological innovation, which shoulder important responsibility and mission in the $\mathrm{Na}$ tional Innovation System. As is expected, the number of R\&D institutions of higher education has increased fast in the past few years. Accurately forecasting the number of R\&D institutions of higher education will provide a reference for the Ministry of Education of the People's Republic of China and the government to make better plans and strategies in advance. However, the effects of related factors on the number of R\&D institutions of higher education are 
TABLe 6: The number of R\&D institutions of higher education from 2011 to 2018 .

\begin{tabular}{lccc}
\hline Year & Number & Year & Number \\
\hline 2011 & 8630 & 2015 & 11732 \\
2012 & 9225 & 2016 & 13062 \\
2013 & 9842 & 2017 & 14971 \\
2014 & 10632 & 2018 & 16280 \\
\hline
\end{tabular}

TABLE 7: Parameter values for five grey models.

\begin{tabular}{lcccccrr}
\hline Parameters & $a$ & $b$ & $\zeta$ & $\gamma$ & $\beta_{1}$ & $\beta_{2}$ & $p$ \\
\hline GM $(1,1)$ & -0.089 & 7.878 & 0.500 & & & & \\
DGM $(1,1)$ & & & & & 1.093 & 8.346 & \\
NGBM $(1,1)$ & -0.117 & 10.590 & 0.500 & -0.126 & & & \\
ONGBM $(1,1)$ & -0.118 & 10.689 & 0.490 & -0.130 & & -0.121 \\
INGBM $(1,1)$ & -0.121 & 10.924 & 0.500 & -0.141 & & 10.941 \\
\hline
\end{tabular}

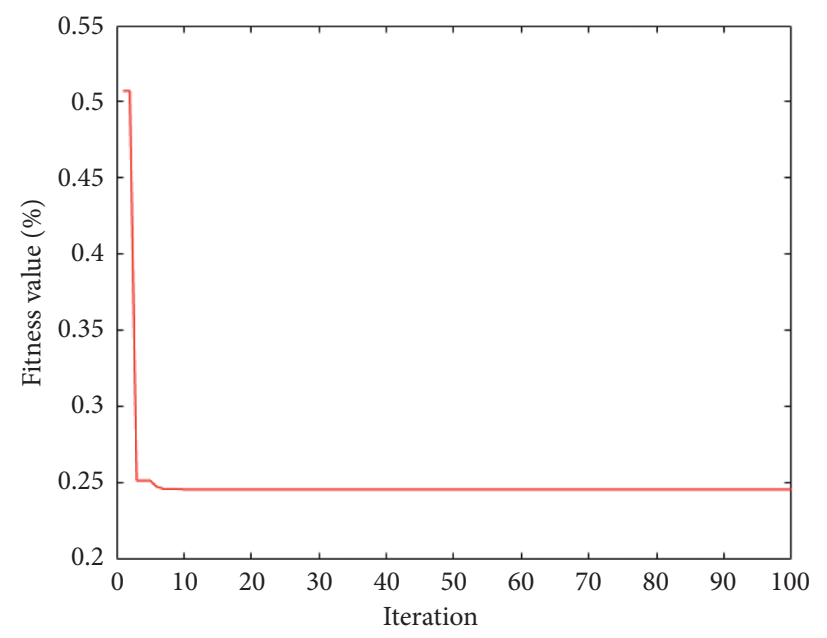

FIGURE 4: The track of searching for the optimal power index by WOA.

quite uncertain, and reliable observations are limited because of China's rapid development, which implies the traditional models (e.g., regression analysis) are not suitable for this case because of the small sample size and uncertain factors. Herein, the proposed model, $\operatorname{INGBM}(1,1)$, is obviously more suitable for this case with few observations.

Empirically, the data collected from China's National Bureau of Statistics of the People's Republic China and listed in Table 6, are divided into two groups, the data from 2011 to 2016 are used to build these five prediction models, and the others are used to assess the accuracy of these models.

Similar to Case 1 and 2, all the parameters in these models are computed and listed in Table 7. Moreover, the track of the power index $\gamma$ using WOA is exhibited in Figure 4.

As a consequence, the simulated and predicted results are shown in Table 8.

In this case, by ignoring the first item of predicted results, it should be known that the RMSE values (see Figure 5) of five grey models are $0.14,0.14,0.04,0.04$, and
0.03 for simulation and are $0.87,0.85,0.39$, and 0.40 for prediction, respectively. Moreover, the MAPE values (see Figure 6 ) of these models are $1.17 \%, 1.18 \%, 0.28 \%, 0.27 \%$, and $0.25 \%$ for simulation and those of models mentioned here are $5.54 \%, 5.46 \%, 2.32 \%, 2.39 \%$, and $1.72 \%$ for prediction, respectively. Therefore, in the simulation period, the proposed model outperforms other grey models with the lowest RMSE value of 0.03 and a MAPE value of $0.27 \%$. The $\operatorname{ONGBM}(1,1)$ model has the following prediction performance with a relatively lower MAPE value of $0.28 \%$. As mentioned in [44], as a proper forecasting method, it performs excellently in simulation and should do well in the prediction stage. By observing Table 8 , it is easy to find that the proposed model is better than other grey models again because of its lower RMSE value of 0.40 and MAPE value of $1.72 \%$. Interestingly, the ONGBM $(1,1)$ is the second better because its MAPE value is a bit higher than that of the $\operatorname{INGBM}(1,1)$, which implies the improved NGBM $(1,1)$ through optimization of background value can be regarded as the alternative 
TABLE 8: Simulated and predicted performance by five grey models using raw data of the number of R\&D institutions of China's higher education.

\begin{tabular}{|c|c|c|c|c|c|}
\hline Actual data & $\mathrm{GM}(1,1)$ & $\operatorname{DGM}(1,1)$ & $\operatorname{NGBM}(1,1)$ & ONGBM $(1,1)$ & INGBM $(1,1)$ \\
\hline \multicolumn{6}{|l|}{8630} \\
\hline 9225 & 9041.86 & 9049.01 & 9224.89 & 9225.38 & 9225.08 \\
\hline 9842 & 9882.75 & 9890.73 & 9820.48 & 9810.66 & 9798.18 \\
\hline 10632 & 10801.83 & 10810.73 & 10692.04 & 10677.82 & 10672.23 \\
\hline 11732 & 11806.39 & 11816.31 & 11760.42 & 11744.56 & 11757.27 \\
\hline 13062 & 12904.37 & 12915.43 & 13011.49 & 12995.95 & 13037.11 \\
\hline RMSE & 0.14 & 0.14 & 0.04 & 0.04 & 0.03 \\
\hline MAPE & 1.17 & 1.18 & 0.28 & 0.27 & 0.25 \\
\hline 14971 & 14104.46 & 14116.78 & 14450.13 & 14436.68 & 14516.89 \\
\hline 16280 & 15416.16 & 15429.88 & 16090.00 & 16080.46 & 16211.58 \\
\hline RMSE & 0.87 & 0.85 & 0.39 & 0.40 & 0.32 \\
\hline MAPE & 5.54 & 5.46 & 2.32 & 2.39 & 1.72 \\
\hline
\end{tabular}

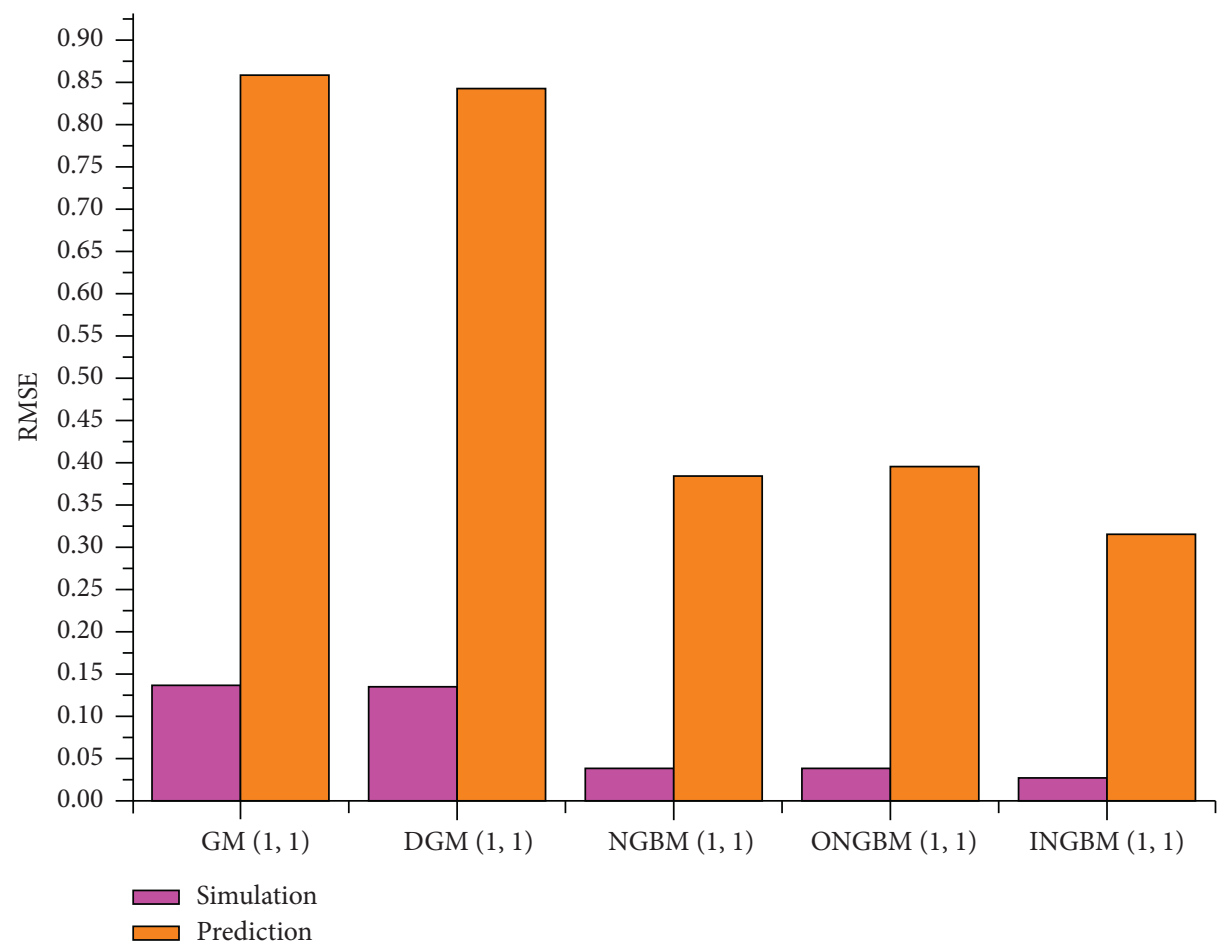

Figure 5: RMSE values generated from five grey system models.

model to predict the number of R\&D institutions of higher education in this paper. In this case, the prediction and fitting errors of all models are not big, which shows that there has no overfitting in the modeling. At the same time, the prediction effect of the nonlinear grey model is better than that of the linear model, which shows that the nonlinear grey model can effectively capture the nonlinear characteristics of the data. Finally, the improved model has the highest accuracy, which indicates that our improvement strategy is effective.

In order to further verify the advantages of WOA, three kinds of intelligent optimizer, grey wolf optimizer (GWO)
[45], particle swarm optimizer (PSO) [46], and ant lion optimizer (ALO) [47], are used for comparison. These four kinds of algorithms are all excellent optimizers with their own characteristics and advantages. The population numbers of the four algorithms are all set to be 100 and the search times to be 100 . The population is initialized 100 times to compare the final MAPE with the corresponding nonlinear parameters and calculate the average time. For the four types of optimization algorithms, the MAPE, and the corresponding nonlinear parameters after running 30 times are shown in Figure 7, and the time consumption is shown in Table 9. 


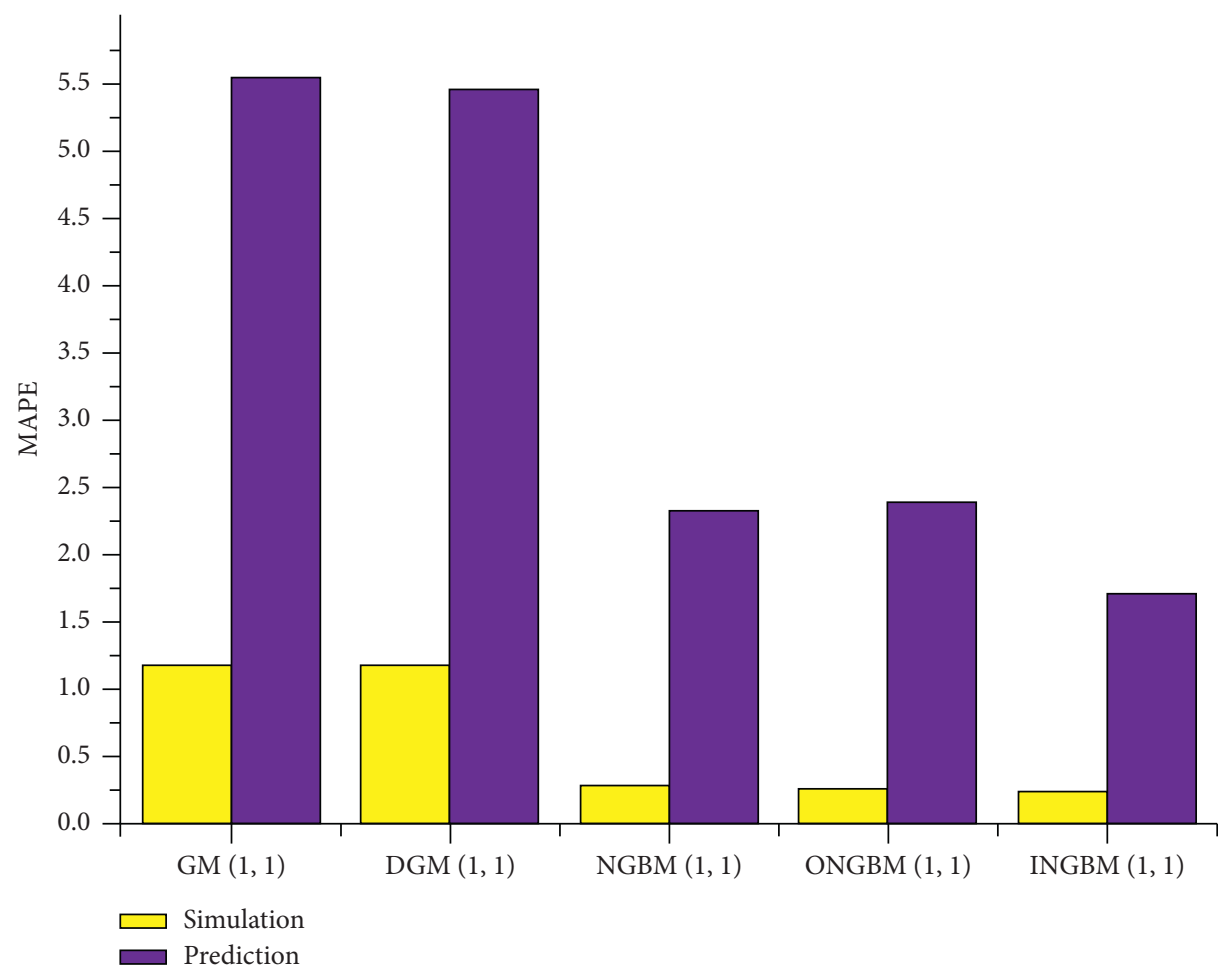

FIgURE 6: MAPE values stemmed from five grey system models.

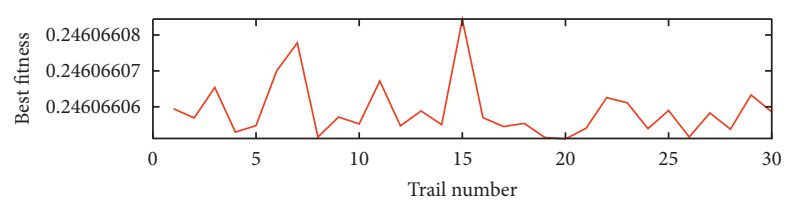

— ALO

(a)

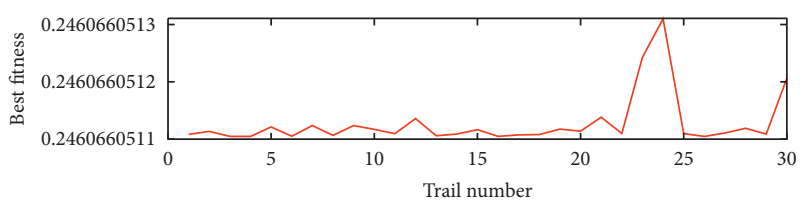

— PSO

(c)

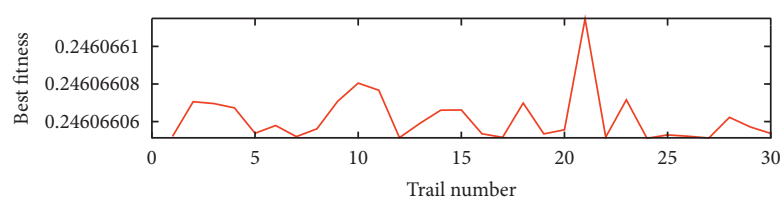

— WOA

(e)

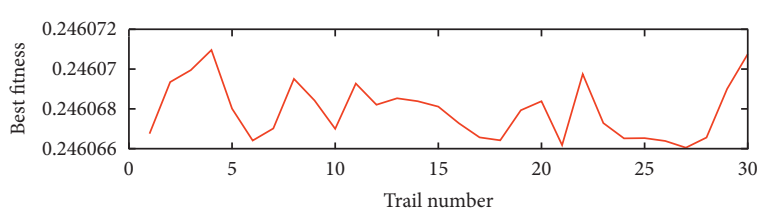

— GWO

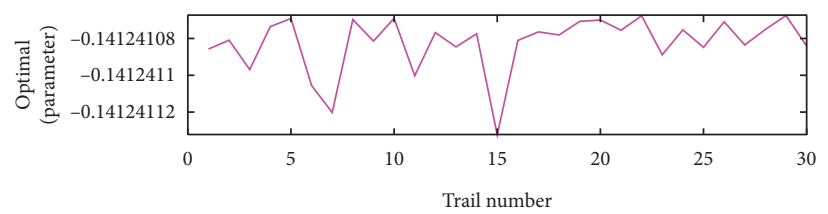

(b)

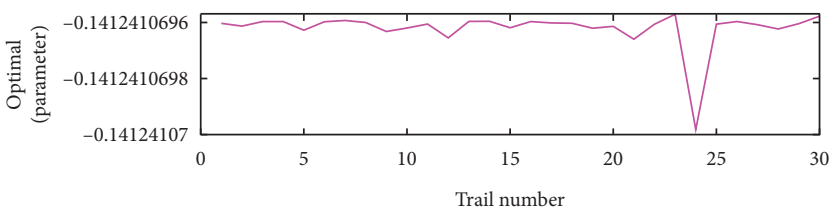

(d)

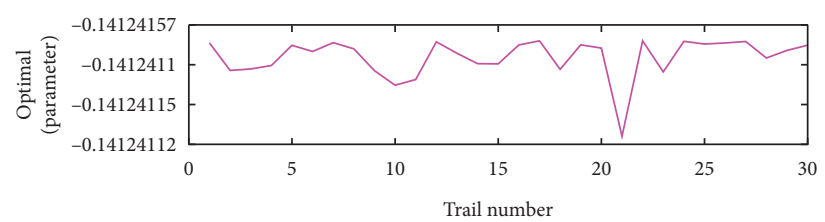

- WOA

(f)

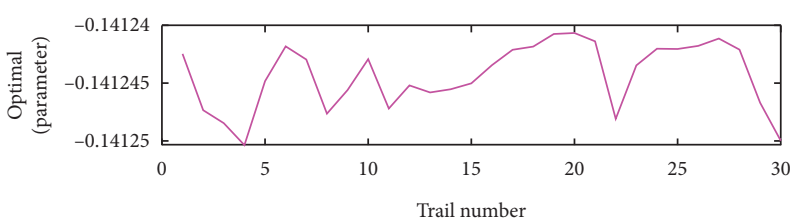

(h)

Figure 7: Performance comparison of four optimizers (WOA, PSO, GWO, and ALO). 
TABLE 9: Average time cost of four optimizers.

\begin{tabular}{lcccc}
\hline Optimizer & GWO & ALO & WOA & PSO \\
\hline Time cost (s) & 6.6671 & 19.5721 & 9.9931 & 16.2616 \\
\hline
\end{tabular}

It can be seen from Figure 7 and Table 9 that the operation of WOA is relatively stable, and the running time of WOA is $9.9931 \mathrm{~s}$, which is relatively small. Overall, the WOA is reasonable as an optimizer.

\section{Conclusion}

This paper aims to further promote the prediction accuracy of the nonlinear grey Bernoulli model $\operatorname{NGBM}(1,1))$, and as a result, the nonlinear grey Bernoulli model with improved parameters, abbreviated as $\operatorname{INGBM}(1,1)$, is proposed. This study does not share the same differential equation as the traditional $\operatorname{NGBM}(1,1)$ model. Instead, the differential equation is transformed into the linear formula. Besides, considering that "misplaced replacement" is the root cause of contradiction when converting the differential equation to the difference equation, the model parameters are optimized to better match these two equations to reduce prediction error. In particular, the whale optimization algorithm is used to automatically determine the optimal power index of INGBM $(1,1)$. Three examples are employed to validate the proposed model's effectiveness by comparing with commonly used grey models. In all cases, the proposed model both outperforms other grey models, implying that the $\operatorname{INGBM}(1,1)$ model can effectively solve the nonlinear problems with a small sample size and provide valuable information for related decisionmakers to make strategies in advance.

Although INGBM $(1,1)$ has a very good effect, there are some limitations that need to be overcome in future work: (1) although the model has a good effect, there may be overfitting in some special cases. (2) More accurate parameter values can be further obtained with multiple optimizers.

\section{Data Availability}

The data used to support the findings of this study are included in this paper.

\section{Conflicts of Interest}

The authors declare that they have no conflicts of interest.

\section{Acknowledgments}

This work was supported by the National Natural Science Foundation of China (11661001) and the Project of Enhancing the Basic Scientific Research Ability of Young and Middle-Aged Teachers in Guangxi Universities (2021KY0740).

\section{References}

[1] J. L. Deng, "Control problems of grey systems," Systems \& Control Letters, vol. 1, no. 5, pp. 288-294, 1982.
[2] S. Ding, K. W. Hipel, and Y.-g. Dang, "Forecasting China's electricity consumption using a new grey prediction model," Energy, vol. 149, pp. 314-328, 2018.

[3] C. Zheng, W. Z. Wu, W. Xie, Q. Li, and T. Zhang, "Forecasting the hydroelectricity consumption of China by using a novel unbiased nonlinear grey Bernoulli model," Journal of Cleaner Production, vol. 278, Article ID 123903, 2020.

[4] C. Liu, T. Lao, W. Z. Wu, and W. Xie, "Application of optimized fractional grey model-based variable background value to predict electricity consumption," Fractals, vol. 141, 2020.

[5] C. Zheng, W. Z. Wu, W. Xie, and Q. Li, "A MFO-based conformable fractional nonhomogeneous grey Bernoulli model for natural gas production and consumption forecasting," Applied Soft Computing, vol. 99, 106891.

[6] Y.-S. Lee and L.-I. Tong, "Forecasting energy consumption using a grey model improved by incorporating genetic programming," Energy Conversion and Management, vol. 52, no. 1, pp. 147-152, 2011.

[7] X. Ma, Z. Liu, and Y. Wang, "Application of a novel nonlinear multivariate grey Bernoulli model to predict the tourist income of China," Journal of Computational and Applied Mathematics, vol. 347, pp. 84-94, 2019.

[8] R. H. Myers and R. H. Myers, Classical and Modern Regressionwith Applications, vol. 2, Duxbury press, Belmont, CA, USA, 1990.

[9] D. M. Bates and D. G. Watts, Nonlinear Regression Analysisand Its Applications, vol. 2, Wiley, New York, NY, USA, 1988.

[10] S. C. Hillmer and G. C. Tiao, "An ARIMA-model-based approach to seasonal adjustment," Journal of the American Statistical Association, vol. 77, no. 377, pp. 63-70, 1982.

[11] T.-M. Choi, Y. Yu, and K.-F. Au, "A hybrid SARIMA wavelet transform method for sales forecasting," Decision Support Systems, vol. 51, no. 1, pp. 130-140, 2011.

[12] B. Scholkopf and A. J. Smola, Learning with Kernels: Support Vector Machines, Regularization, Optimization, and Beyond, MIT Press, Cambridge, MA, USA, 2001.

[13] A. K. Jain, J. Jianchang Mao, and K. M. Mohiuddin, "Artificial neural networks: a tutorial," Computer, vol. 29, no. 3, pp. 31-44, 1996.

[14] N.-m. Xie and S.-f. Liu, "Discrete grey forecasting model and its optimization," Applied Mathematical Modelling, vol. 33, no. 2, pp. 1173-1186, 2009.

[15] L. Wu, S. Liu, L. Yao, S. Yan, and D. Liu, "Grey system model with the fractional order accumulation," Communications in Nonlinear Science and Numerical Simulation, vol. 18, no. 7, pp. 1775-1785, 2013.

[16] T. L. A. Tien, "Research on the grey prediction model GM (1, n)," Applied Mathematics and Computation, vol. 218, no. 9, pp. 4903-4916, 2012.

[17] Z.-X. Wang, Q. Li, and L.-L. Pei, "Grey forecasting method of quarterly hydropower production in China based on a data grouping approach," Applied Mathematical Modelling, vol. 51, pp. 302-316, 2017.

[18] Z.-X. Wang, Q. Li, and L.-L. Pei, “A seasonal GM $(1,1)$ model for forecasting the electricity consumption of the primary economic sectors," Energy, vol. 154, pp. 522-534, 2018.

[19] X. Zeng, L. Shu, S. Yan, Y. Shi, and F. He, "A novel multivariate grey model for forecasting the sequence of ternary interval numbers," Applied Mathematical Modelling, vol. 69, pp. 273-286, 2019.

[20] X. Ma, W. Wu, B. Zeng, Y. Wang, and X. Wu, "The conformable fractional grey system model," ISA Transactions, 2019. 
[21] X. Ma, X. Mei, W. Wu, X. Wu, and B. Zeng, "A novel fractional time delayed grey model with Grey Wolf Optimizer and its applications in forecasting the natural gas and coal consumption in Chongqing China," Energy, vol. 178, pp. 487-507, 2019.

[22] B. Zeng, M. Tong, and X. Ma, "A new-structure grey Verhulst model: development and performance comparison," Applied Mathematical Modelling, vol. 81, pp. 522-537, 2020.

[23] B. Zeng, X. Ma, and M. Zhou, "A new-structure grey Verhulst model for China's tight gas production forecasting," Applied Soft Computing, vol. 96, 2020.

[24] X. S. Yang, "A new metaheuristic bat-inspired algorithm," in Nature Inspired Cooperative Strategies for Optimization, pp. 65-74, 2010.

[25] S. Kirpatrick, C. D. Gelatt, and M. P. Vecchi, "Optimization by simulated annealing," Readings in Computer Vision, pp. 606-615, 1987.

[26] D. E. Goldberg, Genetic Algorithm in Search, Optimization, and Machine Learning, Addison-Wesley Pub. Co., Boston, MA, USA, 1989.

[27] I. C. Trelea, "The particle swarm optimization algorithm: convergence analysis and parameter selection," Information Processing Letters, vol. 85, no. 6, pp. 317-325, 2003.

[28] M. Dorigo and C. Blum, "Ant colony optimization theory: a survey," Theoretical Computer Science, vol. 344, no. 2-3, pp. 243-278, 2005.

[29] B. S. Yldz, A. R. Yildiz, N. Pholdee et al., "The Henry gas solubility optimization algorithm for optimum structural design of automobile brake components," Materials Testing, vol. 62 , no. 3 , pp. $5-25,2020$.

[30] A. R. Yildiz, "A novel hybrid whale-Nelder-Mead algorithm for optimization of design and manufacturing problems," International Journal of Advanced Manufacturing Technology, vol. 105, no. 8, pp. 1-14, 2019.

[31] A. R. Yildiz, S. Mirjalili, S. M. Sait et al., "A new hybrid Harris hawks-Nelder-Mead optimization algorithm for solving design and manufacturing problems," Materials Testing, vol. 8, no. 61, pp. 60-70, 2019.

[32] G.-G. Wang, S. Deb, and Z. Cui, "Monarch butterfly optimization," Neural Computing and Applications, vol. 31, no. 7, pp. 1995-2014, 2019.

[33] F. Hammou and K. Hammouche, "An improved particle swarm optimization algorithm towards IIR system identification," in Proceedings of the 2020 1st International Conference on Communications, Control Systems and Signal Processing (CCSSP), IEEE, El-Oued, Algeria, March 2020.

[34] W. Zhang, R. Xiao, B. Shi, H.-h. Zhu, and Y.-j. Sun, "Forecasting deformation field using correlated grey model updated with time correction slope factor and background value optimization," Engineering Geology, vol. 260, p. 105215, 2019.

[35] H. F. Lu, X. Ma, and K. Huang, "Prediction of offshore wind farm power using a novel two-stage model combining kernelbased nonlinear extension of the Arps decline model with a multi-objective grey wolf optimizer," Renewable and Sustainable Energy Reviews, vol. 127, pp. 1-16, 2020.

[36] W. Wu, X. Ma, B. Zeng, Y. Wang, and W. Cai, "Forecasting short-term renewable energy consumption of China using a novel fractional nonlinear grey Bernoulli model," Renewable Energy, vol. 140, pp. 70-87, 2019.

[37] C.-I. Chen, "Application of the novel nonlinear grey Bernoulli model for forecasting unemployment rate," Chaos, Solitons \& Fractals, vol. 37, no. 1, pp. 278-287, 2008.

[38] W. Z. Wu, T. Zhang, and C. Zheng, "A novel optimized nonlinear Grey Bernoulli Model for forecasting China's
GDP," Complexity, vol. 2019, Article ID 1731262, 10 pages, 2019.

[39] B. Zeng, H. Duan, Y. Bai, and W. Meng, "Forecasting the output of shale gas in China using an unbiased grey model and weakening buffer operator," Energy, vol. 151, pp. 238-249, 2018.

[40] S. Mirjalili and A. Lewis, "The whale optimization algorithm," Advances in Engineering Software, vol. 95, pp. 51-67, 2016.

[41] c. Lewis, Industrial and Business Forecasting Methods, Butterworth Scientific, London, UK, 1982.

[42] L. Q. Zhan and H. J. Shi, "Methods and model of grey modeling for approximation non-homogenous exponential data," Systems Engineering-Theory \& Practice, vol. 33, no. 3, pp. 659-694, 2013.

[43] I.-S. Chen and J.-K. Chen, "Present and future: a trend forecasting and ranking of university types for innovative development from an intellectual capital perspective," Quality \& Quantity, vol. 47, no. 1, pp. 335-352, 2013.

[44] B. Zeng, S. Li, W. Meng, and D. Zhang, "An improved gray prediction model for China's beef consumption forecasting," PLoS One, vol. 14, no. 9, pp. 1-18, 2019.

[45] S. Mirjalili, S. M. Mirjalili, and A. Lewis, "Grey wolf optimizer," Advances in Engineering Software, vol. 69, no. 3, pp. 46-61, 2014.

[46] Z. Rui, Z. Hongguang, S. Songsong et al., "Global optimization of the diesel engine-organic Rankine cycle (ORC) combined system based on particle swarm optimizer (PSO)," Energy Conversion \& Management, vol. 174, pp. 248-259, 2018.

[47] S. Mirjalili, "The ant lion optimizer," Advances in Engineering Software, vol. 83, pp. 80-98, 2015. 